\title{
MicroRNA-381 suppresses cell growth and invasion by targeting the liver receptor homolog-1 in hepatocellular carcinoma
}

\author{
QIANQIAN ZHANG ${ }^{1}$, SHIXING $\mathrm{ZHAO}^{2}, \mathrm{XIAOLI} \mathrm{PANG}^{1}$ and BAORONG CHI ${ }^{1}$ \\ ${ }^{1}$ Department of Hepatobiliary and Pancreatic Diseases, The First Hospital of Jilin University, Changchun, Jilin 130021; \\ ${ }^{2}$ Department of Intensive Care Unit, Affiliated Hospital of Jining Medical University, Jining, Shandong 272000, P.R. China
}

Received September 12, 2015; Accepted October 13, 2015

DOI: 10.3892/or.2015.4491

\begin{abstract}
MicroRNAs (miRs) have emerged as prospective tools for human cancer therapy, including hepatocellular carcinoma (HCC) therapy. Previous studies have suggested that miR-381 functions as oncogenic or tumor-suppressive miRs in other cancer types. However, the role of miR-381 in HCC remains unknown. The present study investigated the expression and functional role of miR-381 in HCC. miR-381 expression was significantly decreased in HCC tissues and cell lines. miR-381 overexpression significantly inhibited HCC cell proliferation and colony formation, induced G0/ G1 cell cycle arrest and suppressed cell invasion. Conversely, suppression of miR-381 showed the opposite effect in HCC cells. Bioinformatics analysis and dual-luciferase reporter assay results showed that miR-381 directly targeted the 3'-untranslated region of liver receptor homolog-1 (LRH-1), and quantitative polymerase chain reaction and western blot analysis results showed that miR-381 negatively modulated LRH-1 expression. Data elucidated that miR-381 directly regulated HCC cell growth and invasion, as well as the Wnt signaling pathways, by targeting LRH-1. Clinical tissue detection data revealed an inverse correlation between miR-381 and LRH-1 expression in HCC tissues, further indicating the functional significance of miR-381-LRH-1 in regulating HCC tumorigenesis. The present study indicates that miR-381 may be a novel tumor suppressor that blocks HCC growth and invasion by targeting LRH-1. The results present novel insights into understanding the molecular mechanism underlying HCC
\end{abstract}

Correspondence to: Dr Baorong Chi, Department of Hepatobiliary and Pancreatic Diseases, The First Hospital of Jilin University, 71 Xinmin Street, Changchun, Jilin 130021, P.R. China

E-mail: chibaorong_jlu@163.com

Abbreviations: miRs, microRNAs; miR-381, microRNA-381; LRH-1, liver receptor homolog-1; 3'-UTR, 3'-untranslated region; HCC, hepatocellular carcinoma

Key words: hepatocellular carcinoma, miR-318, liver receptor homolog-1, cell growth, cell invasion tumorigenesis and provide a future direction to the development of therapeutic interventions for HCC.

\section{Introduction}

Hepatocellular carcinoma (HCC) is one of the most common malignant tumors with increasing morbidity and mortality all over the world (1). Although current therapeutic strategies have improved over the past decades, the treatment outcome and survival rate remain poor because of frequent recurrence and high invasion and metastasis capacity (2-4). One of the obstacles that hampers the development of efficient therapies is the lack of understanding of the precise molecular mechanism underlying HCC development and progression. Thus, a better understanding of the pathological mechanism is important to provide theoretical basis for developing potential and promising therapeutics for $\mathrm{HCC}$.

In recent years, a class of small and non-coding RNAs called microRNAs (miRs) have emerged as prospective tools for cancer therapy, including HCC therapy (5). miRs are negative regulators of gene expression that regulate protein translation by targeting the 3'-untranslated region (UTR) of mRNA $(6,7)$. Studies have indicated that miRs act as potential and useful biomarkers and targets for disease diagnosis, prognosis and treatment $(8,9)$. Other studies have also reported that miRs such as miR-302b (10), miR-1285-3p (11), mR-520g (12) and miR-139 (13) serve as oncogenes or tumor suppressors in HCC. miR-381 is extensively associated with various cancer types $(14,15)$, but its role in HCC has not been comprehensively investigated.

The liver receptor homolog-1 (LRH-1) has been reported as an important regulator in many different cancer types (16). Various studies have uncovered that LRH-1 is involved in regulating embryonic development, ovulation, pregnancy and metabolism (17-22). LRH-1 deficiency blunts intestinal tumorigenesis (23). LRH-1 is elucidated as an oncogene in pancreatic cancer (24), colon cancer (25), gastric cancer (26), breast cancer (27) and ovarian cancer (28). Moreover, LRH-1 is associated with the activation of Wnt signaling pathways (29), which were frequently aberrantly activated in cancers, such as HCC (30). These findings indicate that LRH-1 can serve as a potential molecular target for cancer therapy. Several studies have also attempted to develop an effective antagonist for LRH-1 to treat cancers (31). 
The suppression of LRH-1 in HCC has been demonstrated to induce cell cycle arrest and apoptosis (32), thus indicating its important role in HCC. However, the precise role of LRH-1 in HCC remains largely unknown. In the present report, we demonstrate a targeted relationship between LRH-1 and miR-381. We found that miR-381 was significantly downregulated in HCC cells and tissues. miR-381 overexpression inhibited HCC cell growth and invasion ability, whereas miR-381 suppression displayed an opposite effect. Bioinformatics analysis and dual-luciferase reporter assay results showed that miR-381 directly targeted the 3'-UTR of LRH-1, and quantitative polymerase chain reaction (qPCR) and western blot analysis results showed that miR-381 negatively modulated LRH-1 expression. Data elucidated that miR-381 regulated HCC cell growth and invasion through LRH-1-mediated Wnt signaling. Clinical tissue detection data revealed that an inverse correlation existed between miR-381 and LRH-1 expression, thus further indicating the functional significance of miR-381-LRH-1 in regulating $\mathrm{HCC}$ tumorigenesis. The present study provides evidence that the use of miR-381 to target LRH-1 may be a potential therapeutic strategy for HCC treatment.

\section{Materials and methods}

Tissue specimens. A total of 20 paired malignant $\mathrm{HCC}$ and adjacent non-tumorous liver tissues were obtained from HCC patients undergoing surgical resection in the First Hospital of Jilin University. These patients were first diagnosed without local or systemic treatment before surgical resection. The tissue samples were removed and immediately snap-frozen in liquid nitrogen for further use. Informed consent was obtained from each patient, and the experimental protocol was reviewed and approved by the Institutional Human Experiment and Ethic Committee of the First Hospital of Jilin University.

Cell cultures. Human HCC cell lines including HepG2, SMMC-7721, HuH7, BEL-7402, human normal liver cell line HL-7702 and human embryonic kidney (HEK) 293T cell line were purchased from the American Type Culture Collection (ATCC; Manassas, VA, USA) and were grown in Dulbecco's modified Eagle's medium (DMEM) in a supplement with $10 \%$ heat-inactivated fetal bovine serum (FBS) supplemented with $1 \%$ penicillin-streptomycin (all from Gibco, Grand Island, NY, USA). The cells were cultured in a humidified incubator containing $5 \% \mathrm{CO}_{2}$ atmosphere at $37^{\circ} \mathrm{C}$.

$R N A$ isolation and quantitative polymerase chain reaction $(q P C R)$. Total RNA was extracted by using the miRNeasy Mini kit (Qiagen, Dusseldorf, Germany) according to the manufacturer's recommended method. For mRNA expression analysis, the first-strand cDNA was synthesised by using Moloney murine leukaemia virus (M-MLV) reverse transcriptase (Invitrogen, Carlsbad, CA, USA). The cDNA was then used as templates to amplify LRH-1, cyclin D1, cyclin E1 and MMP9 with specific primers by using SYBR Green Master Mix (Bio-Rad, Hercules, CA, USA). Glyceraldehyde-3-phosphate dehydrogenase (GAPDH) was used as the internal normalisation control. For miRNA expression analysis, the cDNA was obtained using miScript reverse transcription kit and amplified using miScript SYBR Green PCR kit (both from Qiagen) according to the manufacturer's instructions. U6 small nuclear RNA (U6) was used as internal normalization control. The specific primers used in this study were as follows: LRH-1 (forward, 5'-CTGATAGTGGAACTTT TGAA-3' and reverse, 5'-CTTCATTTGGTCATCAACCTT-3'); cyclin D1 (forward, 5'-GTTCGTGGCCTCTAAGATGAAG-3' and reverse, 5'-GTGTTTGCGGATGATCTGTTTG-3'); cyclin E1 (forward, 5'-GCTTCGGCCTTGTATCATTTC-3' and reverse, 5'-CTGTCTCTGTGGGTCTGTATG-3'); MMP9 (forward, 5'-GCCCGACCCGAGCTGACTC-3' and reverse, 5'-TTCAG GGCGAGGACCATAGAGG-3'); GAPDH (forward, 5'-GAAA TCCCATCACCATCTTCCAGG-3' and reverse, 5'-GAGCCC CAGCCTTCTCCATG-3'); miR-381 (forward, 5'-TAATCTGA CTATACAAGGGCAAGCT-3' and reverse, 5'-TATGGTTGT TCTGCTCTCTGTCTC-3'); and U6 (forward, 5'-GCTTCGGC AGCACATATACTAAAAT-3' and reverse, 5'-CGCTTCACGA ATTTGCGTGTCAT-3'). Relative gene expression was analyzed by using the $2^{-\Delta \Delta C t}$ method.

Transfection of HCC cell lines. miR-381 mimics (sense, 5'-UAUACAAGGGCAAGCUCUCUGUTT-3' and antisense, 5'-ACAGAGAGCUUGCCCUUGUCGCTT-3'), scrambled mimics (sense, 5'-UUCUCCGAACGUGUCACGUTT-3' and antisense, 5'-ACGUGACACGUUCGGAGAATT-3'), miR-381 LNA oligonucleotide (inhibitor; 5'-LNAACAGAGAGCUUG CCCUUGUAUA-3') and scrambled LNA (5'-LNACAGUAC UUUUGUGUAGUACAA-3') were purchased from Shanghai GenePharma Co., Ltd. (Shanghai, China). Cells were plated onto 6-well plates and cultured until reaching $80 \%$ confluence. At that time, cells were transiently transfected with miRNA mimics, miR-LNA or their scrambled controls for a final concentration of $20 \mathrm{nM}$ using Lipofectamine 2000 (Invitrogen) according to the supplier's recommended protocol. For LRH-1 overexpression, $4 \mu \mathrm{g}$ of pcDNA3.0/LRH-1 expressing vectors were transiently transfected into HCC cells using Lipofectamine 2000 (Invitrogen) for $48 \mathrm{~h}$. The transfection efficiency was then detected by using western blot analysis.

MTT assay. Cell growth was evaluated by using the MTT method. The HCC cells were briefly seeded in 96-well plates at $1 \times 10^{4}$ cells/well and cultured overnight. The cells were then transfected with miRNA-381 mimics, miR-381 LNA or their scrambled controls and were incubated for 24, 48 and $72 \mathrm{~h}$ followed by discarding the old medium and adding fresh medium containing MTT $(5 \mathrm{mg} / \mathrm{ml}$ in PBS; Sigma, St. Louis, $\mathrm{MO}, \mathrm{USA})$. After culturing for another $4 \mathrm{~h}$ at $37^{\circ} \mathrm{C}$, the formed formazan products were solubilised by adding dimethyl sulfoxide (200 $\mu \mathrm{l} /$ well). The optical density (OD) value was detected at a wavelength of $490 \mathrm{~nm}$ by using a multi-well spectrophotometer (Bio-Tek Instruments, Winooski, VT, USA).

Cell cycle analysis. Cell cycle distribution was detected by using flow cytometry. HCC cells were briefly transfected with miRNA-381 mimics or miR-381 LNA for $48 \mathrm{~h}$. Cells were harvested by trypsinization, washed with PBS and fixed with $70 \%$ ethanol overnight at $4^{\circ} \mathrm{C}$. Approximately $1 \times 10^{6}$ cells were stained with propidium iodide (PI, $100 \mu \mathrm{g} / \mathrm{ml}$; Sigma) solution containing $10 \mu \mathrm{g} / \mathrm{ml}$ of RNase A for $30 \mathrm{~min}$ in the dark. The percentage of cells in each cell cycle phase was then measured by using a FACScan flow cytometer (Becton 

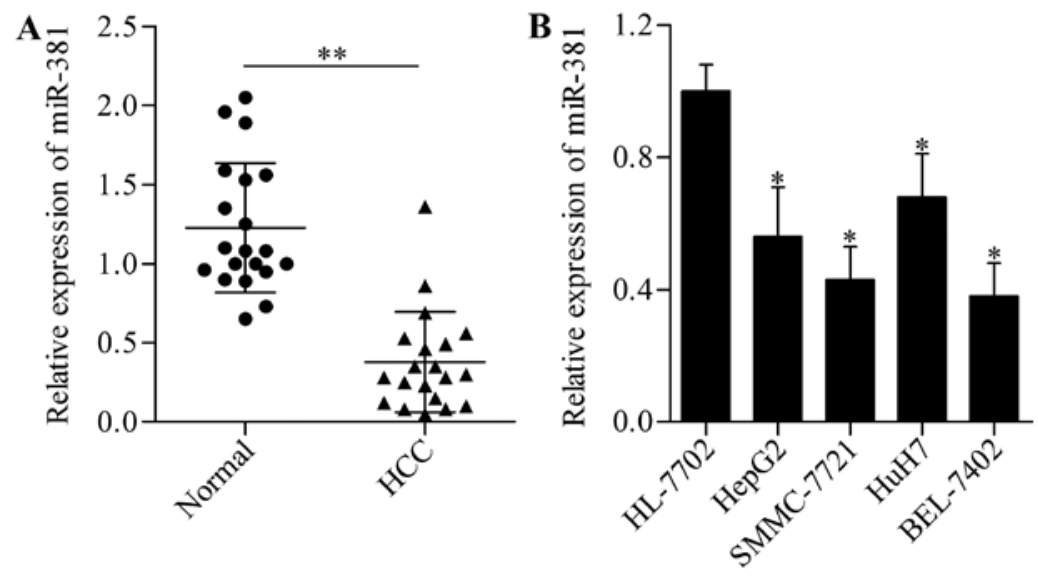

Figure 1. Decreased miR-381 expression in HCC. qPCR analysis of miR-381 expression in HCC tissues (A) and HCC cell lines (B). Non-cancerous counterparts and human normal liver cell line HL-7702 were used as control. ${ }^{* *} \mathrm{P}<0.01$ vs. normal; ${ }^{*} \mathrm{P}<0.05$ vs. HL-7702.

Dickinson, Franklin Lakes, NJ, USA) and analyzed by using ModFit software.

Colony formation assay. HCC cells were cultured in a 6-well plate and transfected with miRNA-381 mimics or miR-381 LNA for $48 \mathrm{~h}$. Thereafter, the transfected cells were re-plated into a 6 -well plate in a growth medium containing $0.3 \%$ noble agar at 200 cells/well to form natural colonies. After 2 weeks, cells were washed with PBS, fixed with $4 \%$ paraformaldehyde and stained with Giemsa (Sigma). The total number of colonies was counted under a microscope.

Cell invasion assay. HCC cells were transfected with miRNA-381 mimics or miR-381 LNA for $48 \mathrm{~h}$ and then starved overnight in serum-free medium. Thereafter, the transfected cells $\left(1 \times 10^{5}\right.$ cells) were seeded into the upper chamber of a 24-well Transwell (Corning Incorporated, Toledo, NY, USA) containing $200 \mu \mathrm{l}$ of serum-free medium. Transwell membrane filter inserts were precoated with Matrigel (BD Biosciences, San Jose, CA, USA). A medium containing 10\% FBS added in the lower chamber was used as a chemoattractant. After incubation for $24 \mathrm{~h}$, the non-invasive cells in the upper chamber were gently removed by a cotton swab. The invasive cells on the lower surface were then fixed, stained and observed under the microscope. The mean number of invaded cells in 5 random fields per membrane was counted and averaged.

Luciferase reporter assay. The targeting relationship between miR-381 and LRH-1 3'-UTR was detected by using a dual-luciferase reporter assay. The cDNA fragments from the 3'-UTR of LRH-1 containing miR-381 binding site were briefly amplified and subcloned into pmirGLO vector (Promega, Madison, WI, USA). HEK293T cells were seeded into 6-well plates at $2 \times 10^{5}$ cells/well for $24 \mathrm{~h}$ and then co-transfected with the $10 \mathrm{ng}$ of pmirGLO-LRH-1 recombinant vectors and $20 \mathrm{nM}$ of miR-381 mimics or scrambled mimics by using Lipofectamine 2000 (Invitrogen). Cells were lysed after $48 \mathrm{~h}$ of transfection, and the luciferase activity was measured by using the dual-luciferase reporter system (Promega). Wnt signaling activity was determined by using Tcf luciferase assays. Cells were briefly co-transfected with TOPFlash firefly luciferase reporter vector (100 ng; Addgene, Cambridge, MA,
USA) and phRL-TK Renilla luciferase vectors (5 ng; Promega) in the presence of miRNA-381 mimics or miR-381 LNA for $48 \mathrm{~h}$. Cells were lysed, and firefly and Renilla luciferase activities were detected by using the dual-luciferase reporter system (Promega). The data were expressed as firefly/Renilla luciferase activity.

Western blot analysis. Cells were lysed by using RIPA lysis buffer, and protein concentrations were measured by using a BCA kit (both from Beyotime Biotechnology, Haimen, China). A total of $25 \mu \mathrm{g}$ proteins were separated by sodium dodecyl sulphate-polyacrylamide gel electrophoresis and then electro-transferred onto a nitrocellulose membrane (Bio-Rad). The membrane was then blocked by $3 \%$ fat-free milk and blotted with primary antibodies (rabbit polyclonal anti-LRH-1, 1:500; rabbit polyclonal anti-GAPDH, 1:800; Santa Cruz Biotechnology Inc., Santa Cruz, CA, USA) at $4^{\circ} \mathrm{C}$ overnight. Thereafter, the membrane was incubated with goat anti-rabbit horseradish peroxidase-conjugated secondary antibodies (1:2,000; Santa Cruz Biotechnology Inc.) for $1 \mathrm{~h}$ at room temperature. The signals of the protein bands were detected by using the enhanced chemiluminescence method (Amersham, Little Chalfont, UK). The signal intensity on the membrane was analysed by Image-Pro Plus 6.0 (Media Cybernetics, Inc., Rockville, MD, USA) and normalized to the control group.

Data analysis. All data were reported as means \pm standard deviation. Statistical analyses were performed with SPSS version 11.5 (SPSS Inc., Chicago, IL, USA) using Student's t-test or one-way analysis of variance. Correlations were assessed by Pearson's correlation test. A p-value of $<0.05$ was set as the level of statistical difference.

\section{Results}

miR-381 downregulation frequently occurred in HCC tissues and cell lines. To investigate the potential function of miR-381 in HCC, we detected first the expression pattern of miR-381 in 20 paired malignant $\mathrm{HCC}$ and adjacent non-tumorous liver tissues by using qPCR. The results showed that miR-381 was frequently and significantly decreased in live HCC samples compared with their peritumor counterparts (Fig. 1A). 
A $\square$ Scrambled-mimics $\square$ miR-381 mimics

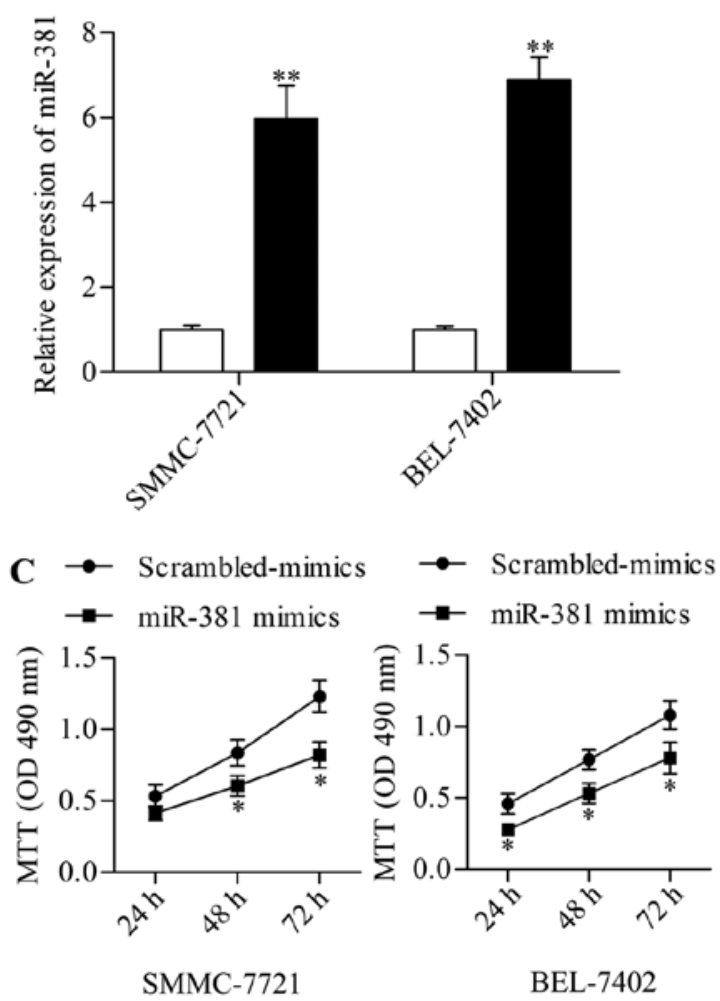

B $\square$ Scrambled-LNA miR-381 LNA
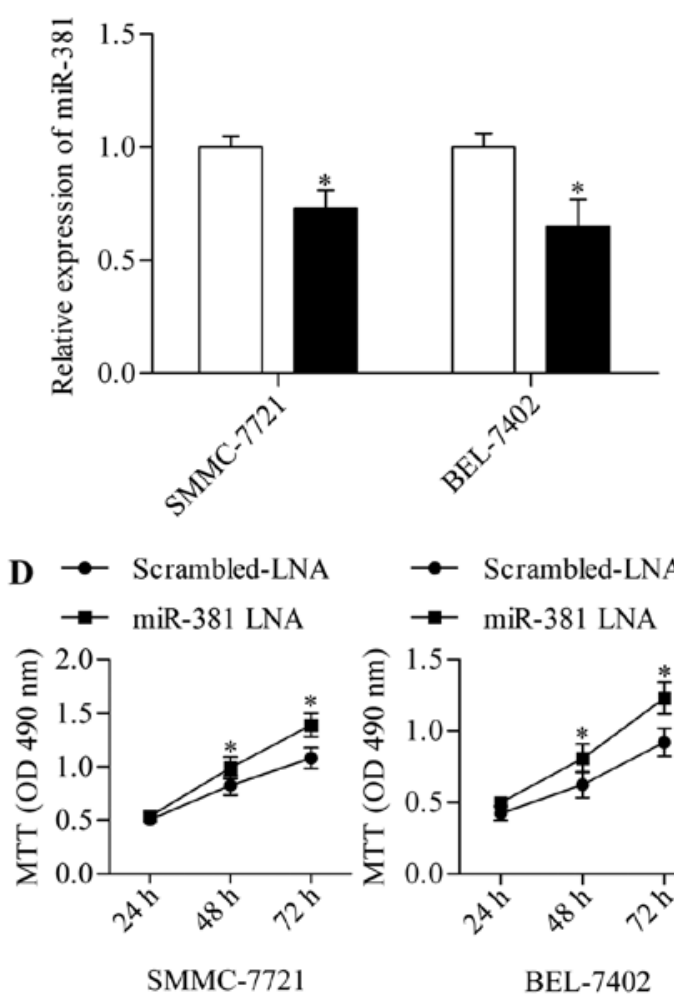

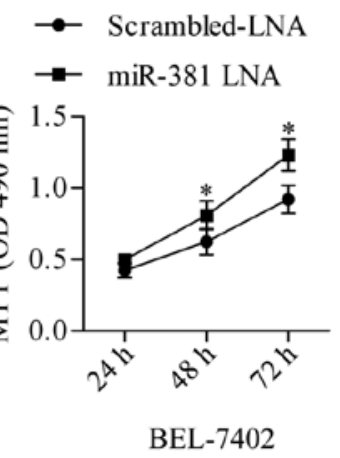

Figure 2. Detection of the role of miR-381 on HCC cell growth. qPCR analysis of miR-381 expression in HCC cells transfected with miR-381 mimics (A) and miR-381 LNA (B) or their scrambled controls. Cells were harvested for qPCR analysis after transfection for $48 \mathrm{~h}$. ${ }^{* *} \mathrm{P}<0.01 \mathrm{vs}$. scrambled mimics; ${ }^{*} \mathrm{P}<0.05 \mathrm{vs}$. scrambled LNA. MTT assay detected the effect of miR-381 mimics (C) and miR-381 LNA (D) transfection on HCC cell growth at 24,48 and $72 \mathrm{~h}$. ${ }^{*} \mathrm{P}<0.05$ vs. scrambled mimics or scrambled LNA.

Furthermore, we detected the expression levels of miR-381 in several HCC cell lines (HepG2, SMMC-7721, HuH7 and BEL-7402) and normal liver cells. This observation was consistent with the results of miR-381 in HCC tissues wherein miR-381 expression was prevalently downregulated compared with human normal liver HL-7702 cells (Fig. 1B). These results indicated that miR-381 may play an important role in $\mathrm{HCC}$ development and progression.

miR-381 inhibits HCC cell growth. To explore the precise function of miR-381 in HCC, we next assessed the effect of miR-381 on HCC growth by using a gain-of-function approach with miR-381 mimics. SMMC-7721 and BEL-7402 cells were transfected with miR-381 mimics followed by detection using MTT assay. The results showed that miR-381 overexpression (Fig. 2A) significantly decreased HCC cell growth (Fig. 2C) in SMMC-7721 and BEL-7402 cells. By contrast, miR-381 suppression (Fig. 2B) significantly promoted HCC cell growth (Fig. 2D). To further verify its effect on cell growth, we examined the effect of miR-381 overexpression on cell cycle progression and found that miR-381 overexpression induced an increase in cell cycle arrest in G0/G1 phase (Fig. 3A). Moreover, the colony-forming capacity of HCC cells was remarkably suppressed by miR-381 overexpression (Fig. 3C). miR-381 suppression by transfection of miR-381 LNA conversely exhibited the opposite effect on cell cycle (Fig. 3B) and colony-forming capacity (Fig. 3D). These results implied that miR-381 functioned as a potential tumor suppressor in HCC.
miR-381 suppresses HCC cell invasion in vitro. To further verify the antitumor effect of miR-381 on HCC, we next examined the effect of miR-381 overexpression or suppression on HCC cell invasion by using Transwell invasion assay. The results showed that miR-381 overexpression significantly decreased the invasion of HCC cells (Fig. 4A), whereas miR-381 suppression further promoted this ability (Fig. 4B).

miR-381 directly targets and mediates $L R H-1$ expression. To uncover the molecular basis of miR-381 in regulating HCC, we sought to screen the putative targets of miR-381 by using bioinformatics analysis. Notably, among those targets, LRH-1 attracted our particular interests because of its important role in various cancers (16). As shown in Fig. 5A, the predicted complementary site of miR-381 was found in the 3'-UTR of LRH-1. We evaluated the dual-luciferase reporter activity by using pmirGLO vector harbouring the wild-type (WT) or mutant (MT) 3'-UTR of LRH-1 to verify whether LRH-1 was the direct target gene of miR-381. Luciferase assay displayed that miR-381 mimics significantly decreased the luciferase activity of pmirGLO-WT LRH-1, whereas miR-381 LNA markedly increased this activity (Fig. 5B). However, neither miR-381 mimics nor miR-381 LNA showed any effects on the luciferase activity of pmirGLO-MT LRH-1 (Fig. 5B). The data indicated that miR-381 directly targeted the predicted complementary site in the 3'-UTR of LRH-1. To further investigate whether miR-381 could regulate endogenous LRH-1 expression, we transfected miR-381 mimics or miR-381 LNA into SMMC-7721 cells and detected LRH-1 expression by using 
A $\square$ Scrambled-mimics $\square$ miR-381 mimics
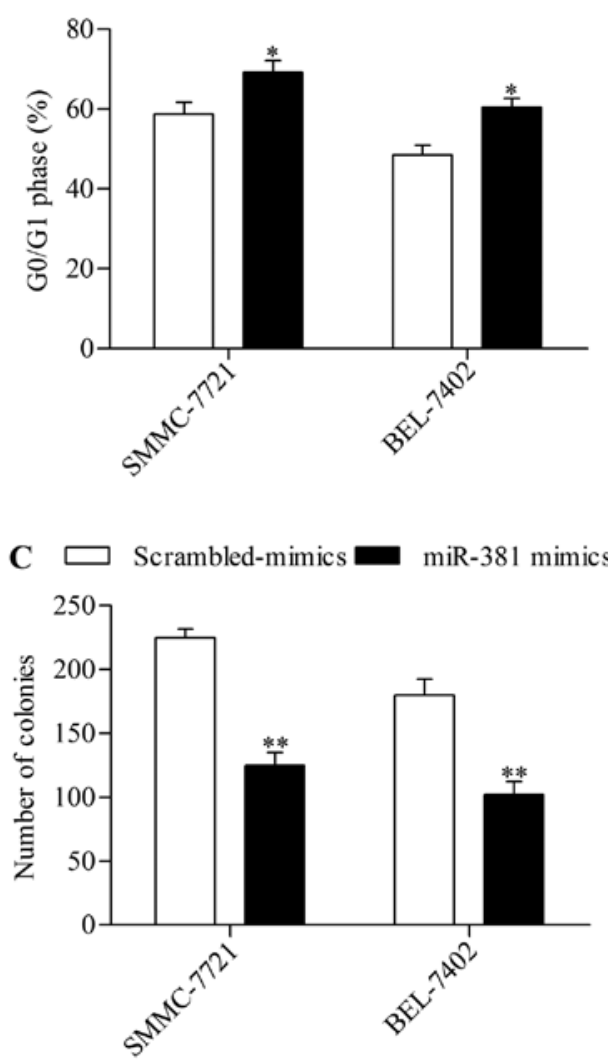
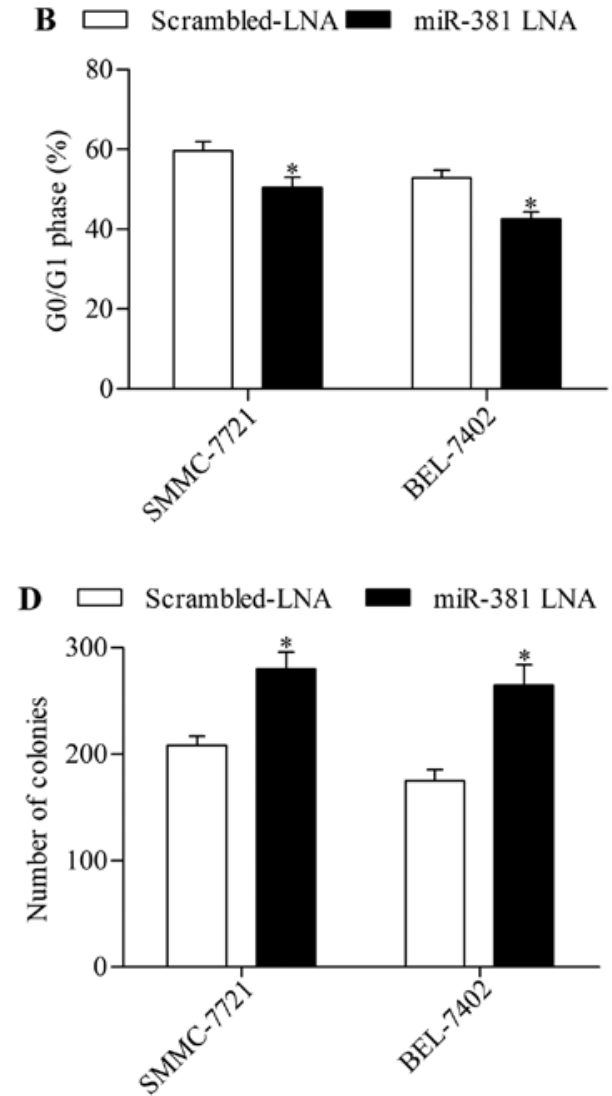

Figure 3. Detection of the role of miR-381 on HCC cell proliferation. The effect of miR-381 mimics (A) and miR-381 LNA (B) on cell cycle distribution was detected by using a cytometry assay. Cells were stained with PI fluorescent dye for flow cytometry assay after transfection for 48 h. ${ }^{*} \mathrm{P}<0.05$ vs. scrambled mimics or scrambled LNA. Effect of miR-381 mimics (C) and miR-381 LNA (D) transfection on the colony-forming capacity of HCC cells. The numbers of colonies were counted by using the Giemsa staining method. ${ }^{* *} \mathrm{P}<0.01$ vs. scrambled mimics; ${ }^{*} \mathrm{P}<0.05$ vs. scrambled LNA.
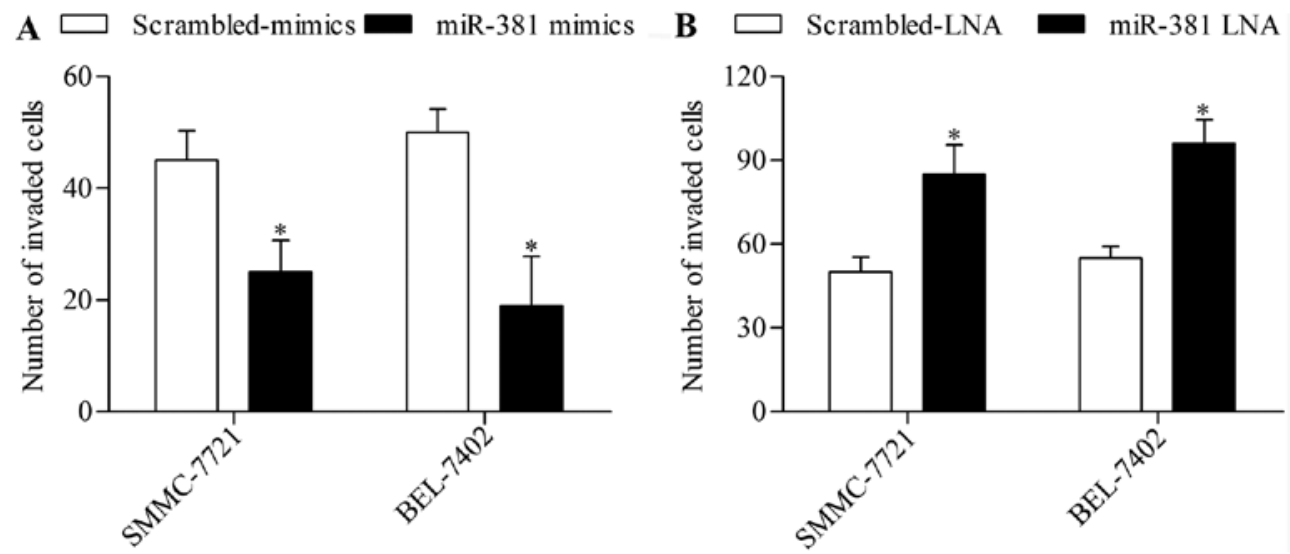

Figure 4. Effect of miR-381 on HCC cell invasion. The effect of miR-381 overexpression (A) or miR-381 suppression (B) on HCC cell invasion ability detected by using Transwell invasion assay. SMMC-7721 and BEL-7402 cells transfected with miR-381 mimics or miR-381 LNA were subjected to Transwell invasion assay. The invaded cells were counted by using the crystal violet staining method after $24 \mathrm{~h}$. $\mathrm{*}<0.05$ vs. scrambled mimics or scrambled LNA.

qPCR and western blot analysis. The results showed that both mRNA and protein expression of LRH-1 was significantly decreased by miR-381 overexpression (Fig. 5C and D). On the contrary, miR-381 suppression further increased LRH-1 expression in SMMC-7221 cells (Fig. 5C and D). Consistent results were obtained by using BEL-7402 cells (data not shown).

miR-381 functions as a tumor suppressor to repress HCC cell growth and invasion through $L R H-1$. We performed a rescue experiment to validate miR-381 functions through LRH-1. HCC cells were co-transfected with miR-381 mimics and LRH-1 expressing vectors without 3'-UTR. Western blot analysis showed that LRH-1 protein expression was significantly restored by LRH-1 expressing vectors transfection even though with miR-381 mimics transfection in SMMC7721 (Fig. 6A) and BEL-7402 (Fig. 6B) cells. MTT assay showed that LRH-1 overexpression significantly reversed the inhibitory effect of miR-381 on HCC cell growth in SMMC- 

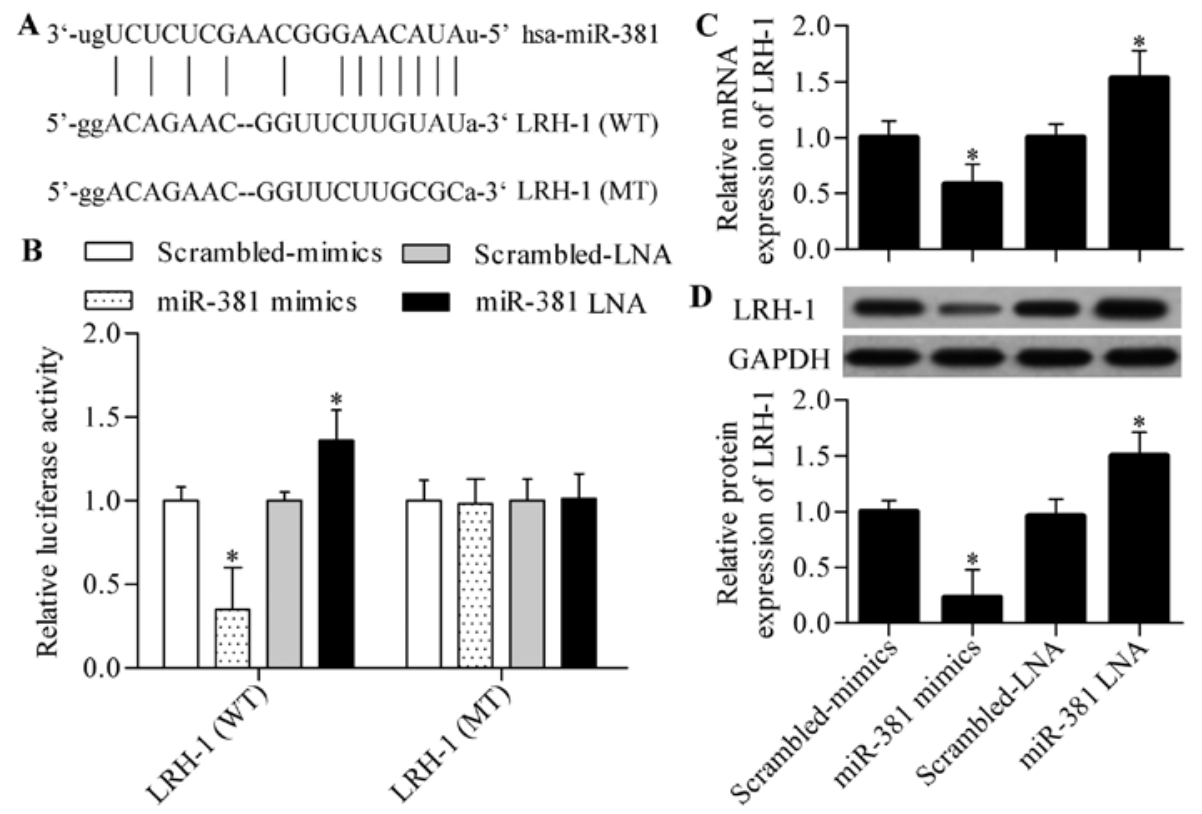

Figure 5. miR-381 targets and regulates LRH-1. (A) Diagram of the predicted miR-381 targeting site in the 3'-UTR of LRH-1. (B) Dual-luciferase reporter assay of the WT and MT LRH-1 3'-UTR reporter constructs in the presence of miR-381 mimics or miR-381 LNA. (C) qPCR analysis of LRH-1 mRNA expression levels in miR-381 mimics or miR-381 LNA transfected SMMC-7721 cells. (D) Western blot analysis detected the LRH-1 protein expression in miR-381 mimics or miR-381 LNA transfected SMMC-7721 cells. Cells were harvested for qPCR or western blot analysis after transfection for $48 \mathrm{~h}$. The relative protein expression of LRH-1 was quantitated by using Image-Pro Plus 6.0 and normalized to GAPDH. ${ }^{*} \mathrm{P}<0.05$ vs. scrambled mimics or scrambled LNA.
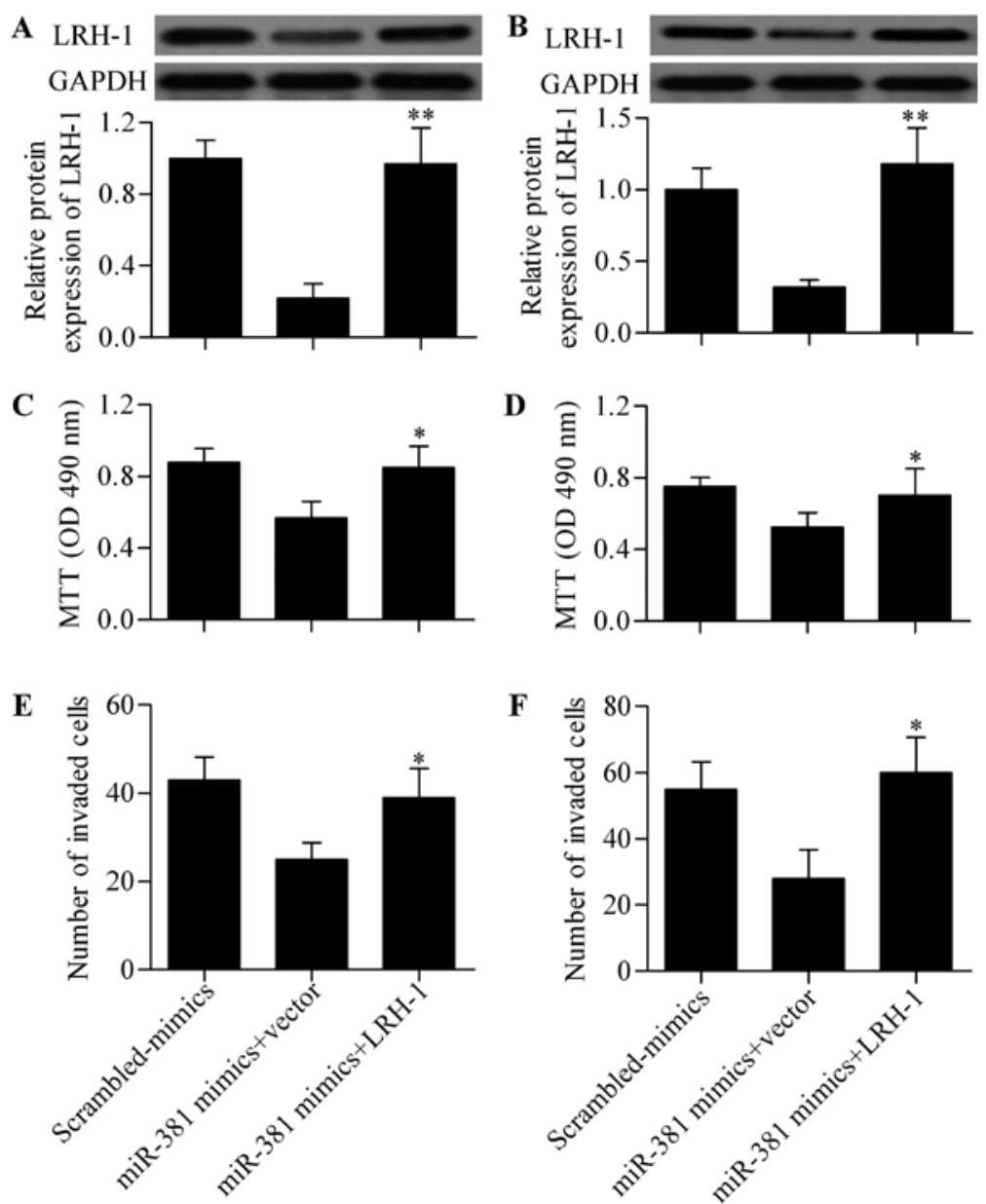

Figure 6. miR-381 inhibits HCC cell growth and invasion through LRH-1. Western blot analysis detected the LRH-1 protein expression in SMMC-7721 (A) and BEL-7402 (B) cells co-transfected with miR-381 mimics and LRH-1 expressing vectors for $48 \mathrm{~h}$. The relative protein expression of LRH-1 was quantitated using Image-Pro Plus 6.0. * $\mathrm{P}<0.01$ vs. miR-381 mimics + vector. MTT assay detected the effect of LRH-1 overexpression on miR-381-induced cell growth arrest in SMMC-7721 (C) and BEL-7402 (D) cells. "P<0.05 vs. miR-381 mimics + vector. The effect of LRH-1 expressing vectors and miR-381 mimic cotransfection on cell invasion in SMMC-7721 (E) and BEL-7402 (F) cells. " $\mathrm{P}<0.05$ vs. miR-381 mimics + vector. 

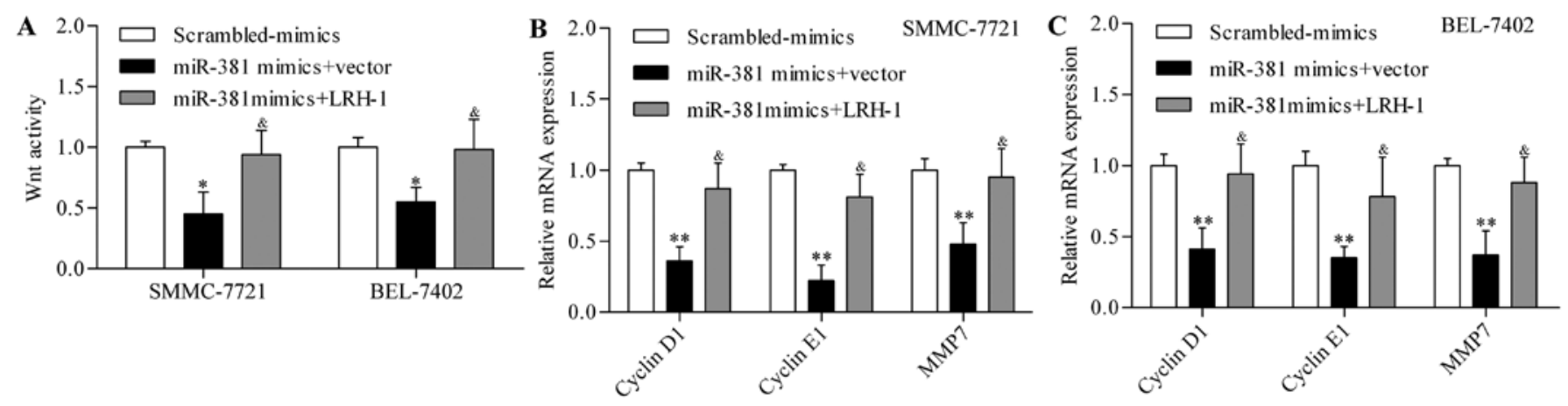

Figure 7. miR-381 negatively regulates Wnt signaling pathway through LRH-1. (A) Tcf-dependent TOPFlash reporter activity assay detected Wnt signalling activity. "P<0.05 vs. scrambled mimics; ${ }^{\circledR} \mathrm{P}<0.05$ vs. miR-381 mimics + vector. qPCR analysis of cyclin D1, cyclin E1 and MMP9 mRNA expression levels in SMMC-7721 (B) and BEL-7402 (C) cells. HCC cells were co-transfected with miR-381 mimics and LRH-1 expressing vectors for $48 \mathrm{~h}$. ${ }^{* *} \mathrm{P}<0.01 \mathrm{vs}$. scrambled mimics; ${ }^{\&} \mathrm{P}<0.05$ vs. miR-381 mimics + vector.
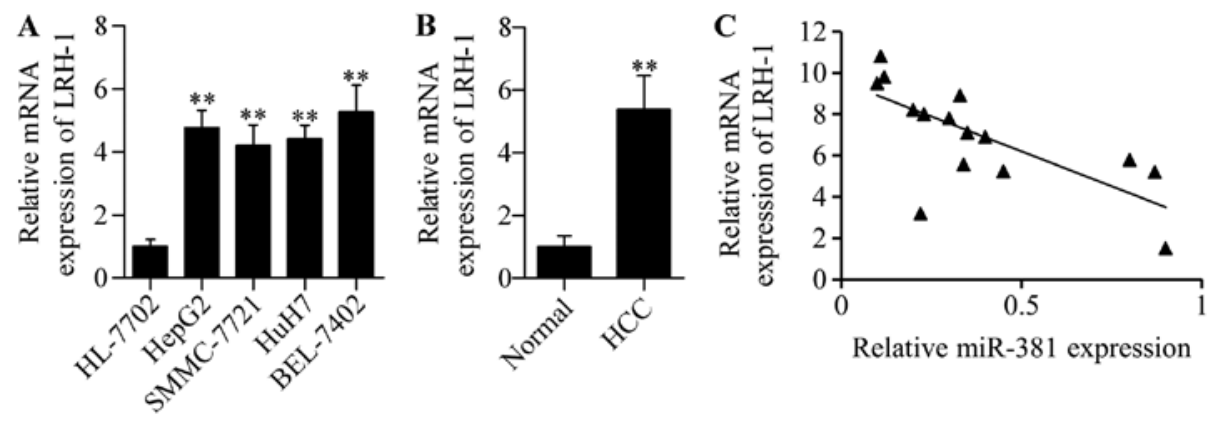

Figure 8. miR-381 inversely correlates with LRH-1. qPCR analysis of LRH-1 expression in HCC cell lines (A) and HCC tissues (B). ${ }^{* *} \mathrm{P}<0.01$ vs. HL-7702 cells or normal tissues. (C) miR-381 expression levels inversely correlated with LRH-1 expression in HCC tissues. R=-0.6996, $\mathrm{P}<0.001$.

7721 (Fig. 6C) and BEL-7402 (Fig. 6D) cells. The results further showed that the decreased cell invasion ability induced by miR-381 overexpression was also remarkably reversed by LRH-1 overexpression (Fig. 6E and F). These results indicated that miR-381 exerted its antitumor effect by inhibiting LRH-1.

miR-381 negatively regulates the Wnt signaling pathway through LRH-1 in HCC cells. To further elucidate the underlying mechanism of miR-381 in regulating $\mathrm{HCC}$, we investigated the role of miR-381 in regulating the downstream signaling pathway of LRH-1. Previous studies have reported that LRH-1 can promote Wnt signalling pathways (29), which were frequently aberrantly activated in cancers including HCC (30). Wnt signaling activity was determined by using Tcf-dependent TOPFlash reporter activity assay, and the results showed that miR-381 overexpression significantly downregulated Wnt signaling activity in SMMC-7721 and BEL-7402 cells (Fig. 7A). To further verify the regulatory effect of miR-318 on Wnt signaling pathway, we next detected the transcriptional activity of the Wnt signaling pathway. The results showed that the expression of Wnt target genes, including cyclin D1, cyclin E1 and MMP9, were significantly decreased by miR-381 overexpression in SMMC-7721 (Fig. 7B) and BEL-7402 (Fig. 7C) cells. Moreover, the restoration of LRH-1 expression significantly reversed the inhibitory effect of miR-381 overexpression on Wnt signaling activity (Fig. 7). These results indicated that miR-381 regulated Wnt signaling pathway through LRH-1.
miR-381 expression level inversely correlates with LRH-1 expression in HCC tissues. To further explore the functional significance of miR-381 in HCC, we detected its association with LRH-1 in HCC tissues. We found that LRH-1 mRNA was highly expressed in HCC cells (Fig. 8A) and HCC tissues (Fig. 8B). Furthermore, correlation analysis showed that miR-381 expression was inversely associated with LRH-1 expression in HCC tissues (Fig. 8C). These results indicated that dysregulated miR-381 expression may contribute to increased LRH-1 expression and HCC development and progression.

\section{Discussion}

In the present study, we found that low miR-381 expression and high LRH-1 expression co-occurred in HCC tissues and cell lines showing an inverse correlation relationship. The study demonstrated a direct targeted relationship between miR-381 and LRH-1, in which miR-381 negatively regulated LRH-1 expression. The decreased miR-381 expression in HCC may contribute to the highly overexpressed LRH-1 and thus promoted tumor development and progression. This study provides novel insights into the molecular mechanism underlying the pathogenesis of HCC malignancy.

The role of LRH-1 has been extensively studied during the past decades. LRH-1 is required for embryonic development (33) and for maintaining the pluripotency of embryonic stem cells (34). LRH-1 is essential for pregnancy, and a lack of 
LRH-1 results in infertility (19,35). Moreover,LRH-1 regulated bile acid biosynthesis and glucose sensing processes $(20,22)$. However, the deregulated LRH-1 contributed to disease occurrences such as cancer. The most studied cancer type is breast cancer, wherein LRH-1 was overexpressed, which was associated with aromatase production (36-38). Annicotte et al reported that LRH-1 was an estrogen receptor target gene that exhibited potential oncogenic effects during tumorigenesis of breast cancer (39). LRH-1 was also capable of regulating the estrogen receptor expression and estrogen receptor-mediated target gene expression that promoted breast cancer cell proliferation $(27,40,41)$. LRH-1 overexpression promoted breast cancer cell migration and invasion (42). LRH-1 can directly target the promoter region of cyclindependent kinase inhibitor p21 and inhibit p21 expression leading to breast cancer cell proliferation (43). Benod et al found that LRH-1 was overexpressed in human pancreatic cancer cells, and LRH-1 knockdown significantly repressed pancreatic cancer cell proliferation (24). Silencing of LRH-1 inhibited colon cancer cell proliferation and induced G0/ G1 cycle arrest (25). LRH-1 was highly expressed in gastric cancer tissues, and LRH-1 overexpression promoted cell proliferation of gastric cancer (26). All these studies pointed out the oncogenic role of LRH-1. The present results of our study also demonstrated that LRH-1 was overexpressed in HCC tissues and cell lines, and LRH-1 downregulation by miR-381 impeded the tumorigenic potential of HCC cells, including cell growth arrest and decreased invasion ability. These results were consistent with the findings of Wang et al, who reported that LRH-1 knockdown inhibited cell growth and induced cell apoptosis of HCC cells (32). Interestingly, it was reported that LRH-1 could facilitate hepatitis B virus DNA replication and gene transcription, which promoted the risk for HCC tumorigenesis (44). Overall, these findings supported the notion that LRH-1 can be used as a potential and promising molecular target for HCC prevention and treatment.

Several studies have been performed to develop a novel antagonist for LRH-1 $(45,46)$. Corzo et al reported that a small molecule repressor of LRH-1 and SR1848 exhibited anti-proliferation activity against LRH-1-expressing cancer cells by inactivating LRH-1 (31). miRs have received particular attention for cancer detection and treatment in recent years because of their negative regulator effect on gene expression (47). A variety of miRs have been found to be potential molecular target for HCC treatment by targeting oncogenes or tumor suppressors. For instance, Wang et al reported that miR-302b inhibited HCC cell invasion and metastasis by inhibiting AKT2 expression (10). miR-99b accelerated HCC cell metastasis by suppressing claudin 11 expression (48). Recent studies have demonstrated that miR-381 plays an important role in various cancer types. For example, Chen et al revealed that miR-381 inhibited renal cancer cell proliferation and sensitised renal cancer cells to chemotherapeutics by inhibiting WEE1 $(14,49)$. miR-381 overexpression suppressed multidrug resistance of cancer cells (50). In lung cancers, miR-381 overexpression inhibits cancer cell migration and invasion by inhibiting the inhibitor of differentiation 1 (15). miR-381 was downregulated in prostate cancer, and the lower expression level was correlated with higher incidence of metastatic events (51). Consistent with these studies, the present study demonstrated that miR-381 was also significantly downregulated in HCC, and miR-381 overexpression markedly suppressed HCC cell growth and invasion. The critical role of miR-381 in HCC was shown for the first time. miR-381 exerted antitumor effects by targeting LRH-1, thereby implying that LRH-1 was one of the miR-381 targets. miR-381 exhibited different functions due to regulating different targets. Tian et al reported that miR-381 functioned as an oncomiR that promoted glioma growth by inhibiting leucine-rich repeat $\mathrm{C} 4$ expression $(52,53)$. miR-381 inhibition sensitised glioma cells to temozolomide by upregulating neurofilament light polypeptide (54). Moreover, Papp et al elucidated that miR-381 may promote epithelioid sarcoma by inhibiting SWI/SNF-related, matrix-associated, actin-dependent regulator of chromatin, subfamily b, member 1 (55). These conflicting findings implicated that miR-381 may exert different functions by regulating different targets in different cells or conditions.

LRH-1 is a co-activator of Wnt signaling pathway that synergistically promoted the transcriptional activity of Wnt signaling pathway by inducing cyclin E1, cyclin D1 and c-myc expression (29). Wnt signaling pathway is frequently aberrantly activated in HCC, which is associated with increased cancer cell growth and metastasis (30). LRH-1 overexpression promoted gastric adenocarcinoma cell proliferation by inducing cyclin E1 (26). LRH-1 knockdown inhibited cyclin E1, cyclin D1 and c-myc expression in pancreatic cancer cells (24). In the present study, downregulating LRH-1 by miR-381 inactivated Wnt signaling and repressed the expression of target genes, including cyclin E1, cyclin D1 and MMP9. Most importantly, restoration of LRH-1 expression reversed the inhibitory effect of miR-381 on the Wnt signaling pathway, which may explain why miR-381 overexpression impeded HCC cell growth and invasion.

To date, the specific miRs that target and regulate LRH-1 have not been well recognized and investigated. A recent study elucidated that miR-451 directly targets and suppresses LRH-1 expression in osteosarcoma cells, thereby inhibiting cancer cell proliferation (56). In the present study, miR-381 targeted and regulated LRH-1 expression in HCC cells. These findings raised the possibility that inhibiting LRH-1 by specific miR to treat and prevent cancers in which LRH-1 functioned as an oncogene. The present results therefore suggest that miR-381 may be a novel tumor suppressor that blocks HCC growth and invasion by targeting LRH-1 and inhibits the LRH-1-mediated Wnt signaling pathway. The present study highlighted the functional significance of miR-381-LRH-1 in HCC, providing novel insights into the development of future therapeutic interventions for HCC.

\section{References}

1. Siegel RL, Miller KD and Jemal A: Cancer statistics, 2015. CA Cancer J Clin 65: 5-29, 2015.

2. Okuda K: Early recognition of hepatocellular carcinoma. Hepatology 6: 729-738, 1986.

3. Kuo KL, Stenehjem D, Albright F, Ray S and Brixner D: Treatment patterns and outcomes in patients with hepatocellular carcinoma stratified by stage-guided treatment categories. J Natl Compr Canc Netw 13: 987-994, 2015. 
4. Song S, Nam SW, Bae SH, Kim JD, Jang JW, Song MJ, Lee SW, Kim HY, Lee YJ, Chun HJ, et al: Outcome of transarterial chemoembolization-based multi-modal treatment in patients with unresectable hepatocellular carcinoma. World J Gastroenterol 21: 2395-2404, 2015.

5. Song Y, Wang F, Huang Q, Cao Y, Zhao Y and Yang C: MicroRNAs contribute to hepatocellular carcinoma. Mini Rev Med Chem 15: 459-466, 2015.

6. Bartel DP: MicroRNAs: Genomics, biogenesis, mechanism, and function. Cell 116: 281-297, 2004.

7. Winter J, Jung S, Keller S, Gregory RI and Diederichs S: Many roads to maturity: microRNA biogenesis pathways and their regulation. Nat Cell Biol 11: 228-234, 2009.

8. Mendell JT and Olson EN: MicroRNAs in stress signaling and human disease. Cell 148: 1172-1187, 2012.

9. Ranganathan K and Sivasankar V: MicroRNAs - Biology and clinical applications. J Oral Maxillofac Pathol 18: 229-234 2014.

10. Wang L, Yao J, Zhang X, Guo B, Le X, Cubberly M, Li Z, Nan K, Song T and Huang C: miRNA-302b suppresses human hepatocellular carcinoma by targeting AKT2. Mol Cancer Res 12 190-202, 2014

11. Liu J, Yan J, Zhou C, Ma Q, Jin Q and Yang Z: miR-1285-3p acts as a potential tumor suppressor miRNA via downregulating JUN expression in hepatocellular carcinoma. Tumour Biol 36 219-225, 2015

12. Kan H, Guo W, Huang Y and Liu D: MicroRNA-520g induces epithelial-mesenchymal transition and promotes metastasis of hepatocellular carcinoma by targeting SMAD7. FEBS Lett 589: 102-109, 2015

13. Li T, Yin J, Yuan L, Wang S, Yang L, Du X and Lu J: Downregulation of microRNA-139 is associated with hepatocellular carcinoma risk and short-term survival. Oncol Rep 31: 1699-1706, 2014

14. Chen B, Duan L, Yin G, Tan J and Jiang X: Simultaneously expressed miR-424 and miR-381 synergistically suppress the proliferation and survival of renal cancer cells - Cdc2 activity is up-regulated by targeting WEE1. Clinics (Sao Paulo) 68 : 825-833, 2013.

15. Rothschild SI, Tschan MP, Jaggi R, Fey MF, Gugger M and Gautschi O: MicroRNA-381 represses ID1 and is deregulated in lung adenocarcinoma. J Thorac Oncol 7: 1069-1077, 2012.

16. Nadolny $\mathrm{C}$ and Dong X: Liver receptor homolog-1 (LRH-1): A potential therapeutic target for cancer. Cancer Biol Ther 16: 997-1004, 2015.

17. Paré JF, Malenfant D, Courtemanche C, Jacob-Wagner M, Roy S, Allard D and Bélanger L: The fetoprotein transcription factor (FTF) gene is essential to embryogenesis and cholesterol homeostasis and is regulated by a DR4 element. J Biol Chem 279: 21206-21216, 2004.

18. Duggavathi R, Volle DH, Mataki C, Antal MC, Messaddeq N, Auwerx J, Murphy BD and Schoonjans K: Liver receptor homolog 1 is essential for ovulation. Genes Dev 22: 1871-1876, 2008.

19. Zhang C, Large MJ, Duggavathi R, DeMayo FJ, Lydon JP, Schoonjans K, Kovanci E and Murphy BD: Liver receptor homolog-1 is essential for pregnancy. Nat Med 19: 1061-1066, 2013.

20. Lu TT, Makishima M, Repa JJ, Schoonjans K, Kerr TA, Auwerx J and Mangelsdorf DJ: Molecular basis for feedback regulation of bile acid synthesis by nuclear receptors. Mol Cell 6: 507-515, 2000.

21. Mataki C, Magnier BC, Houten SM, Annicotte JS, Argmann C, Thomas C, Overmars H, Kulik W, Metzger D, Auwerx J, et al: Compromised intestinal lipid absorption in mice with a liver-specific deficiency of liver receptor homolog 1 . Mol Cell Biol 27: 8330-8339, 2007

22. Oosterveer MH, Mataki C, Yamamoto H, Harach T, Moullan N, van Dijk TH, Ayuso E, Bosch F, Postic C, Groen AK, et al: LRH-1-dependent glucose sensing determines intermediary metabolism in liver. J Clin Invest 122: 2817-2826, 2012.

23. Schoonjans K, Dubuquoy L, Mebis J, Fayard E, Wendling O, Haby C, Geboes K and Auwerx J: Liver receptor homolog 1 contributes to intestinal tumor formation through effects on cell cycle and inflammation. Proc Natl Acad Sci USA 102 2058-2062, 2005

24. Benod C, Vinogradova MV, Jouravel N, Kim GE, Fletterick RJ and Sablin EP: Nuclear receptor liver receptor homologue 1 (LRH-1) regulates pancreatic cancer cell growth and proliferation. Proc Natl Acad Sci USA 108: 16927-16931, 2011.
25. Bayrer JR, Mukkamala S, Sablin EP, Webb P and Fletterick RJ: Silencing LRH-1 in colon cancer cell lines impairs proliferation and alters gene expression programs. Proc Natl Acad Sci USA 112: 2467-2472, 2015

26. Wang SL, Zheng DZ, Lan FH, Deng XJ, Zeng J, Li CJ, Wang R and Zhu ZY: Increased expression of hLRH-1 in human gastric cancer and its implication in tumorigenesis. Mol Cell Biochem 308: 93-100, 2008.

27. Chand AL, Wijayakumara DD, Knower KC, Herridge KA, Howard TL, Lazarus KA and Clyne CD: The orphan nuclear receptor LRH-1 and ER $\alpha$ activate GREB1 expression to induce breast cancer cell proliferation. PLoS One 7: e31593, 2012.

28. Chand AL, Pathirage N, Lazarus K, Chu S, Drummond AE, Fuller PJ and Clyne CD: Liver receptor homologue-1 expression in ovarian epithelial and granulosa cell tumours. Steroids 78 : 700-706, 2013

29. Botrugno OA, Fayard E, Annicotte JS, Haby C, Brennan T, Wendling O, Tanaka T, Kodama T, Thomas W, Auwerx J, et al: Synergy between LRH-1 and beta-catenin induces G1 cyclin-mediated cell proliferation. Mol Cell 15: 499-509, 2004.

30. Pez F, Lopez A, Kim M, Wands JR, Caron de Fromentel C and Merle P: Wnt signaling and hepatocarcinogenesis: Molecular targets for the development of innovative anticancer drugs. J Hepatol 59: 1107-1117, 2013.

31. Corzo CA, Mari Y, Chang MR, Khan T, Kuruvilla D, Nuhant P, Kumar N, West GM, Duckett DR, Roush WR, et al: Antiproliferation activity of a small molecule repressor of liver receptor homolog 1. Mol Pharmacol 87: 296-304, 2015.

32. Wang S, Lan F, Huang L, Dong L, Zhu Z, Li Z, Xie Y and Fu J: Suppression of hLRH-1 mediated by a DNA vector-based RNA interference results in cell cycle arrest and induction of apoptosis in hepatocellular carcinoma cell BEL-7402. Biochem Biophys Res Commun 333: 917-924, 2005.

33. Gu P, Goodwin B, Chung AC, Xu X, Wheeler DA, Price RR, Galardi C, Peng L, Latour AM, Koller BH, et al: Orphan nuclear receptor LRH-1 is required to maintain Oct4 expression at the epiblast stage of embryonic development. Mol Cell Biol 25: 3492-3505, 2005

34. Kelly VR and Hammer GD: LRH-1 and Nanog regulate Dax1 transcription in mouse embryonic stem cells. Mol Cell Endocrinol 332: 116-124, 2011.

35. Gerrits H, Paradé MC, Koonen-Reemst AM, Bakker NE, Timmer-Hellings L, Sollewijn Gelpke MD and Gossen JA: Reversible infertility in a liver receptor homologue-1 (LRH-1)-knockdown mouse model. Reprod Fertil Dev 26: 293-306, 2014

36. Zhou J, Suzuki T, Kovacic A, Saito R, Miki Y, Ishida T, Moriya T, Simpson ER, Sasano H and Clyne CD: Interactions between prostaglandin $\mathrm{E}(2)$, liver receptor homologue-1, and aromatase in breast cancer. Cancer Res 65: 657-663, 2005

37. Bouchard MF, Taniguchi $\mathrm{H}$ and Viger RS: Protein kinase A-dependent synergism between GATA factors and the nuclear receptor, liver receptor homolog-1, regulates human aromatase (CYP19) PII promoter activity in breast cancer cells Endocrinology 146: 4905-4916, 2005.

38. Lanzino M, Maris P, Sirianni R, Barone I, Casaburi I, Chimento A, Giordano C, Morelli C, Sisci D, Rizza P, et al: DAX-1, as an androgen-target gene, inhibits aromatase expression: A novel mechanism blocking estrogen-dependent breast cancer cell proliferation. Cell Death Dis 4: e724, 2013.

39. Annicotte JS, Chavey C, Servant N, Teyssier J, Bardin A, Licznar A, Badia E, Pujol P, Vignon F, Maudelonde T, et al: The nuclear receptor liver receptor homolog-1 is an estrogen receptor target gene. Oncogene 24: 8167-8175, 2005.

40. Thiruchelvam PT, Lai CF, Hua H, Thomas RS, Hurtado A, Hudson W, Bayly AR, Kyle FJ, Periyasamy M, Photiou A, et al: The liver receptor homolog-1 regulates estrogen receptor expression in breast cancer cells. Breast Cancer Res Treat 127: 385-396, 2011.

41. Lai CF, Flach KD, Alexi X, Fox SP, Ottaviani S, Thiruchelvam PT, Kyle FJ, Thomas RS, Launchbury R, Hua H, et al: Co-regulated gene expression by oestrogen receptor $\alpha$ and liver receptor homolog-1 is a feature of the oestrogen response in breast cancer cells. Nucleic Acids Res 41: 10228-10240, 2013.

42. Chand AL, Herridge KA, Thompson EW and Clyne CD: The orphan nuclear receptor LRH-1 promotes breast cancer motility and invasion. Endocr Relat Cancer 17: 965-975, 2010.

43. Bianco S, Jangal M, Garneau D and Gévry N: LRH-1 controls proliferation in breast tumor cells by regulating CDKN1A gene expression. Oncogene 34: 4509-4518, 2015. 
44. Cai YN, Zhou Q, Kong YY, Li M, Viollet B, Xie YH and Wang Y: LRH-1/hB1F and HNF1 synergistically up-regulate hepatitis B virus gene transcription and DNA replication. Cell Res 13: 451-458, 2003.

45. Rey J, Hu H, Kyle F, Lai CF, Buluwela L, Coombes RC, Ortlund EA, Ali S, Snyder JP and Barrett AG: Discovery of a new class of liver receptor homolog-1 (LRH-1) antagonists: Virtual screening, synthesis and biological evaluation. ChemMedChem 7: 1909-1914, 2012.

46. Benod C, Carlsson J, Uthayaruban R, Hwang P, Irwin JJ, Doak AK, Shoichet BK, Sablin EP and Fletterick RJ: Structure-based discovery of antagonists of nuclear receptor LRH-1. J Biol Chem 288: 19830-19844, 2013.

47. Anwar SL and Lehmann U: MicroRNAs: Emerging novel clinical biomarkers for hepatocellular carcinomas. J Clin Med 4: $1631-1650,2015$

48. Yang J, Liu X, Yuan X and Wang Z: miR-99b promotes metastasis of hepatocellular carcinoma through inhibition of claudin 11 expression and may serve as a prognostic marker. Oncol Rep 34: $1415-1423,2015$

49. Chen B, Duan L, Yin G, Tan J and Jiang X: miR-381, a novel intrinsic WEE1 inhibitor, sensitizes renal cancer cells to 5-FU by up-regulation of $\mathrm{Cdc} 2$ activities in 786-O. J Chemother 25 229-238, 2013

50. Xu Y, Ohms SJ, Li Z, Wang Q, Gong G, Hu Y, Mao Z, Shannon MF and Fan JY: Changes in the expression of miR-381 and miR-495 are inversely associated with the expression of the MDR1 gene and development of multi-drug resistance. PLoS One 8: e82062, 2013.
51. Formosa A, Markert EK, Lena AM, Italiano D, Finazzi-Agró E, Levine AJ, Bernardini S, Garabadgiu AV, Melino G and Candi E: MicroRNAs, miR-154, miR-299-5p, miR-376a, miR-376c miR-377, miR-381, miR-487b, miR-485-3p, miR-495 and miR-654-3p, mapped to the 14q32.31 locus, regulate proliferation, apoptosis, migration and invasion in metastatic prostate cancer cells. Oncogene 33: 5173-5182, 2014.

52. Tang H, Liu X, Wang Z, She X, Zeng X, Deng M, Liao Q, Guo X, Wang R, Li X, et al: Interaction of hsa-miR-381 and glioma suppressor LRRC4 is involved in glioma growth. Brain Res 1390: 21-32, 2011.

53. Tang H, Wang Z, Liu Q, Liu X, Wu M and Li G: Disturbing miR-182 and -381 inhibits BRD7 transcription and glioma growth by directly targeting LRRC4. PLoS One 9: e84146, 2014.

54. Wang Z, Yang J, Xu G, Wang W, Liu C, Yang H, Yu Z, Lei Q, Xiao L, Xiong J, et al: Targeting miR-381-NEFL axis sensitizes glioblastoma cells to temozolomide by regulating stemness factors and multidrug resistance factors. Oncotarget 6:3147-3164, 2015.

55. Papp G, Krausz T, Stricker TP, Szendrői M and Sápi Z: SMARCB1 expression in epithelioid sarcoma is regulated by miR-206, miR-381, and miR-671-5p on both mRNA and protein levels. Genes Chromosomes Cancer 53: 168-176, 2014.

56. Li Z, Wu S, Lv S, Wang H, Wang Y and Guo Q: Suppression of liver receptor homolog-1 by microRNA-451 represses the proliferation of osteosarcoma cells. Biochem Biophys Res Commun 461: 450-455, 2015. 